\title{
Different Faces of Sinonasal Mass Lesions
}

https://doi.org/10.47210/bjohns.2020.v28i3.288

Devika T, ${ }^{1}$ Shubha P Bhat, ${ }^{2}$ Vadisha Srinivas Bhat, ${ }^{1}$ Rajeshwary Aroor, ${ }^{1}$ Satheesh Kumar Bhandary B, ${ }^{1}$ Gautham MK, ${ }^{1}$ Samatha KJ

Introduction

ABSTRACT

Mass in the nasal cavity presents with a wide range of symptoms, when a presumptive diagnosis is often made with the help of imaging and endoscopy. This study focussed on correlating clinical diagnosis with the histopathological diagnosis so that appropriate treatment can be offered to improve the quality of life of the patient.

\section{Materials and Methods}

The study included 120 cases who presented with symptoms and signs of mass in the nasal cavity, undergoing surgery or diagnostic biopsy. They were evaluated with a detailed history and clinical examination, diagnostic nasal endoscopy, and relevant radiological investigations. Histopathological examination of the biopsy of the excised specimen was performed by Haematoxylin and Eosin stain. Special stains and Immunohistochemistry (IHC) were performed whenever indicated. The clinical diagnosis was correlated with histopathological diagnosis.

$\underline{\text { Results }}$

Nasal obstruction was the most frequent symptom followed by nasal discharge. Non-neoplastic lesions made up $85 \%$ of cases, while $16 \%$ of cases were proved as neoplastic lesions. Among neoplastic lesions, 7\% were benign, and 9\% were malignant. The inflammatory polyp was the most common non-neoplastic lesion. Fischer's exact test showed a correlation between clinical diagnosis and histopathological diagnosis. Non-neoplastic lesions were common in the 4th decade of life; benign lesions were common in the 3rd decade of life, while malignant lesions were common in the 5th decade of life.

\section{Conclusion}

Sinonasal masses present with overlapping clinical features, and sometimes the definite diagnosis is possible only by histopathological examination of the specimen. However, in the presence of characteristic clinical features, accurate clinical diagnosis is possible in most cases, and appropriate treatment can be performed without delay, pending histopathological examination..

$\underline{\text { Keywords }}$

Mass,Sinonasal; Presentation; Histopathology

$\mathrm{T}$ he nasal cavity hosts many diseases that present with common symptoms like nasal obstruction, nasal discharge, epistaxis, or anosmia. ${ }^{1}$ Sinonasal mass lesions may be congenital, inflammatory, or benign or malignant neoplasm. Congenital mass can be intranasal or extra nasal dermoid, glioma or encephalocele. The Inflammatory polyp develops from the lining mucosa of the nose, ethmoid, and maxillary sinuses. It shows connective tissue edema, with infiltration of plasma, neutrophils, and eosinophils. Benign tumors may arise from the osteocartilaginous skeleton, respiratory mucosa or glandular, vascular, and neural structures within the sinuses. Malignant tumors of the nasal cavity are relatively uncommon, representing $3 \%$ of head and neck malignancy and less than $1 \%$ of all malignancies. ${ }^{2}$ Sinonasal malignancies with coexisting infection lead to delay in diagnosis as early tumors can mimic sinusitis.

Sinonasal tumors may be large on presentation and

1 - Department of ENT, K S Hegde Medical Academy,

Mangalore

2 - Department of Pathology, K S Hegde Medical Academy, Mangalore

\section{Corresponding author:}

Dr Vadisha Srinivas Bhat

email: bvadish@yahoo.co.in 
invade structures of orbit and skull base or extend into nearby foramina or pathways of least resistance. Due to the complexity of these tumors, imaging may require a combination of a Computed Tomography (CT) and Magnetic Resonance Imaging (MRI). The primary goal of imaging is to identify the nature of the primary disease, determine its degree of aggression, delineate the local extent of the tumor, characterize perineural spread and orbital invasion, and evaluate skull base and lymph node involvement. CT scan is essential for assessing bone involvement or destruction. MRI characterizes soft tissue involvement.

This study aims to analyze and correlate the clinical and histopathological characteristics of sinonasal masses and to identify the occurrence of various neoplastic and non-neoplastic lesions in different age groups.

\section{Materials and Methods}

This observational study was conducted in the department of ENT, in a tertiary care hospital of Mangaluru between January 2017 and January 2019. Approval for the study was obtained from the Institutional Ethics Committee. The study included 120 cases who presented with symptoms and signs of mass in the nasal cavity. They were evaluated with a detailed history and clinical examination, including diagnostic nasal endoscopy after written informed consent. Radiological investigations like CT scan or MRI were performed wherever indicated. Patients undergoing diagnostic biopsy or surgical excision were included in the study. Histopathological examination of the specimen was performed by Haematoxylin and Eosin stain. Special stains like Gomori's methenamine silver (GMS) and Periodic Acid Schiff (PAS) were performed as and when required. Immunohistochemistry (IHC) was performed whenever indicated. The correlation between clinical diagnosis and histopathological diagnosis was carried out using Fischer's exact test. The occurrence of benign and malignant lesions and their age and gender distribution were carried out by the Chi-square test.

\section{Results}

The study population included one hundred and twenty patients with a mass in the nasal cavity and paranasal sinuses. Out of them, $80(67 \%)$ were males, and 40 $(33 \%)$ were females, with a ratio of $2: 1$ showing a male predominance. Most of the patients belonged to the age group of 51-60 years, 27 (23\%) patients being in this group, followed by 24 patients $(20 \%)$ in $31-40$ years. The oldest patient was 79 years year old, the youngest being seven years. Nasal obstruction was the most frequent symptom in our study $(71.7 \%)$, followed by nasal discharge (20.8\%), epistaxis (14\%), and headache $(14 \%)$. Eight patients $(7 \%)$ had eye symptoms, and 6 (5\%) had disturbed smell sensation.

Out of 120 patients, $79(66 \%)$ were clinically diagnosed as sinonasal polyp. The following common clinical diagnosis was fungal sinusitis in $11(9.2 \%)$ patients. Six patients $(5 \%)$ were diagnosed as nasopharyngeal carcinoma and an equal number as inverted papilloma. Four cases $(3.3 \%)$ each of angiomatous polyp and granulomatous disease, $3(2.5 \%)$ of maxillary sinus malignancy, $2(1.6 \%)$ each of rhinosporidiosis, olfactory neuroblastoma and Angiofibroma and 1 (0.83\%) frontoethmoidal mucocele were the other clinical diagnoses, based on the clinical examination, nasal endoscopy, and imaging studies.

On histopathological examination, 101 (84\%) were non-neoplastic lesions, while $19(16 \%)$ of cases were neoplastic lesions ( $7 \%$ benign and $9 \%$ malignant). Among the non- neoplastic lesions, 74 (61.7\%) were inflammatory polyp. (Table I)

Benign neoplasm consisted of $5(4.2 \%)$ Inverted papilloma, 2 (1.6\%) Angiofibroma, and 1(0.83\%) Schwannoma. Out of the eleven malignant neoplasms, 5 were nasopharyngeal carcinoma, 2 Olfactoryneuroblastoma, and one each of pleomorphic sarcoma, squamous cell carcinoma, malignant round cell tumor of the maxillary sinus. One case of adenocarcinoma of the nasal septum was a surprise for the clinicians. (Table II)

We correlated the clinical diagnosis with the histopathological diagnosis. Out of the 79 cases clinically diagnosed with sinonasal polyp, 69 (87.3\%) were histopathologically confirmed as Inflammatory polyp, $3(3.7 \%)$ as allergic nasal polyp, $2(2.5 \%)$ each as angiomatous polyp, fungal sinusitis, and inverted 
Table I: Non-neoplastic lesions

\begin{tabular}{|c|c|c|}
\hline HISTOPATHOLOGY & NUMBER (N=120) & PERCENTAGE (\%) \\
\hline Inflammatory polyp & $\mathbf{7 4}$ & $\mathbf{1 0}$ \\
\hline Fungal sinusitis & 4 & 3.3 \\
\hline Angiomatous polyp & 3 & 2.5 \\
\hline Allergic nasal polyp & 2 & 1.6 \\
\hline Rhinosporidiosis & 2 & 1.6 \\
\hline Pyogenic granuloma & 1 & 0.83 \\
\hline Tuberculosis & 1 & 0.83 \\
\hline Rhinoscleroma & 1 & 0.83 \\
\hline Rosai Dorfman's disease & 1 & 0.83 \\
\hline
\end{tabular}

papilloma and $1(1.2 \%)$ as adenocarcinoma. Even though the diagnosis of 78 out of 79 patients remains within the inflammatory pathology, one of the cases turned out to be a malignant condition, which required appropriate treatment. Eleven cases were clinically and radiologically diagnosed as fungal sinusitis, of which 8 (72.8\%) were histopathologically confirmed as invasive fungal sinusitis (Mucormycosis), and 2 (18.2\%) as noninvasive (aspergillosis) fungal sinusitis.

Six cases were clinically diagnosed as Nasopharyngeal carcinoma, of which 5 (83.3\%) were histopathologically confirmed as nasopharyngeal carcinoma and $1(16.7 \%)$ as schwannoma, which is a benign lesion. Three cases were clinically diagnosed as malignancy of maxillary sinus, of which one each 1(33.3 $\%$ ) were histopathologically diagnosed as squamous cell carcinoma, pleomorphic sarcoma, and malignant round cell tumor of the maxillary sinus. All three were confirmed to be malignant conditions. Clinical diagnosis of Angiofibroma and olfactory neuroblastoma were confirmed as histopathologically confirmed.

Six cases were clinically diagnosed as Inverted papilloma based on the clinical examination and imaging, of which $3(50 \%)$ were histopathologically diagnosed as inverted papilloma, 2(33.3\%) as an inflammatory polyp, and $1(16.7 \%)$ as Angiofibroma of the nasal septum. In two cases, large and long-standing polyp leading to changes on the surface mimicked inverted papilloma.

Table II: Malignant neoplasm

\begin{tabular}{|c|c|c|}
\hline HISTOPATHOLOGY & NUMBER $(\mathrm{N}=120)$ & PERCENTAGE (\%) \\
\hline Nasopharyngeal carcinoma & 5 & 4.2 \\
\hline Olfactory Neuroblastoma & 2 & 0.83 \\
\hline Adenocarcinoma of Septum & 1 & 0.83 \\
\hline Pleomorphic sarcoma & 1 & 0.83 \\
\hline Squamous cell carcinoma & 1 & 0.83 \\
\hline Malignant Round cell tumour & 1 & 2 \\
\hline
\end{tabular}


Four cases were clinically diagnosed as angiomatous polyp, of which $2(50 \%)$ were histopathologically diagnosed as pyogenic granuloma, one $(25 \%)$ each as angiomatous polyp and inflammatory polyp. The four cases clinically diagnosed as granulomatous diseases were histopathologically diagnosed as Rosai Dorfman's disease, tuberculosis, Rhinoscleroma, and inflammatory polyp.

The clinico-histopathological correlation in our study was $92 \%$. Fischer's exact test was significant across all age groups $(\mathrm{p}=0.01)$. Inflammatory lesions were common in the second decade of life. Benign lesions were common in the fifth decade, and malignant lesions were common in the elderly. However, there was no statistically significant difference in the occurrence of benign and malignant lesions in males and females $(\mathrm{p}=0.061)$.

\section{Discussion}

The mass lesions in the nasal cavity and paranasal sinuses have different histological features. It is essential to differentiate non-neoplastic from malignant neoplastic lesions to decide the modality of treatment and for timely intervention. The neoplastic and non- neoplastic lesions may present with overlapping clinical features, which makes it difficult to differentiate them clinically. ${ }^{1}$ Histopathological examination of the biopsy of the nasal mass is performed before complete excision when the clinical diagnosis mandates histological diagnosis for further treatment. In some cases, Histopathological examination is performed after complete surgical excision of the mass for confirmation of the diagnosis and to decide about the additional course of treatment. Due to the common clinical presentation of inflammatory lesions, the histopathological examination is essential for proper management. ${ }^{3}$ There are instances where malignant tumors of nose and sinuses show polypoidal features and can present like simple nasal polyps. ${ }^{4}$

Our study involved 120 cases of nasal mass, with a male predominance, with a male to female ratio of 2:1. Clinical studies on nasal mass lesions have shown that nasal mass lesions have a male preponderance. ${ }^{5}$ Bakari et al. in a study from Nigeria found a predominance of females showing male to female ratio at 1: 1.2 and observed a higher incidence in the third decade of life. ${ }^{6}$
Nasal obstruction was the most common presentation $(71.6 \%)$ in our study, followed by nasal discharge $(20.8 \%)$, epistaxis and headache (11.6\% each). Other symptoms were eye-related symptoms, neck nodes, and anosmia. In general, bilateral or unilateral nasal obstruction has been reported as a main presenting symptom, which is seen in more than $80 \%$ of the cases. ${ }^{4,7}$ Clinically, most of our cases had a diagnosis of inflammatory, non-neoplastic lesions (78\%), out of which $66 \%$ were inflammatory polyp. Benign lesions consisted of $\%$ of the study population, and $11 \%$ were diagnosed as malignant lesions.

On histopathological examination, neoplastic lesions made up $85 \%$ of cases, while $16 \%$ of cases were neoplastic lesions in our study, which is similar to most of the reports worldwide. The inflammatory polyp was the most common non-neoplastic lesion in our study. Among neoplastic lesions, 7\% were benign, and 9\% were malignant lesions. Gupta et al. studied 92 patients of sinonasal mass lesions and found that 74 cases were non-neoplastic, and 18 were neoplastic, comprising 12 benign and six malignant lesions. 8 The inflammatory polyp was the most common histopathological diagnosis in our study; this was the commonest in most of the studies, with a share of $60-85 \%$ of the total cases. ${ }^{4,9}$

The clinico-histopathological correlation in our study was $92 \%$. Interestingly, most of the studies done on the clinicopathological relationship of nasal mass lesions correlated by more than $95 \% .^{10,11}$ Imaging modalities like CT scan points out the severity of the disease, involvement of adjacent structures and guides in diagnosis, planning the management. But we believe histopathology is the gold standard for diagnosis as one percent change in the clinical diagnosis, which makes a benign lesion into a malignant lesion, will change the plan of treatment. Sarkar et al. reported a series of six cases of nasal polyposis where the histopathological diagnosis was olfactory neuroblastoma, adenoid cystic carcinoma, basal cell adenocarcinoma, mucosal melanoma, primitive neuroectodermal tumor, and aspergilloma. ${ }^{12}$ In our study, one of the 79 cases clinically diagnosed as nasal polyp had an underlying adenocarcinoma, which could have been missed if the histopathological examination was not performed. However, in the presence of characteristic clinical 
features, a clinical diagnosis may be accurate, and necessary treatment may be initiated with pending histopathological confirmation, to avoid delay in initiation of treatment. Also, in some unique cases like Angiofibroma, a biopsy is contraindicated, and the diagnosis is purely based on clinical and radiological findings. Histopathological examination is possible only on completion of the surgical treatment.

The present study showed that inflammatory lesions were common in the second decade of life. Benign lesions were common in the fifth decade, and malignant lesions were common in the elderly. The inflammatory polyp is a common disease of the second and third decades of life. ${ }^{3}$ Angiofibromais mostly seen in adolescent males. Only one case of Angiofibroma in our study was in the second decade. Olfactory neuroblastoma has a mean age of presentation of 30 years. $^{7}$ In our research, olfactory neuroblastoma was seen in elderly males. ${ }^{7}$ In our study, one case of squamous cell carcinoma was observed in the sixth decade. We did not find any statistically significant difference in the occurrence of benign and malignant lesions in males and females.

\section{Conclusion}

The mass lesions in nasal and paranasal sinuses have diverse etiology. They present with overlapping clinical features, and sometimes it is challenging to differentiate neoplastic and non-neoplastic lesions. Due to the similarities in the initial presentation of non-neoplastic and neoplastic lesions, the diagnosis of a malignant lesion may be missed or delayed, which otherwise requires early intervention. In the presence of characteristic clinical features, accurate clinical diagnosis is possible in most cases, and appropriate surgical treatment can be performed without delay. However, histopathology of the resected specimen is essential to confirm the diagnosis.

\section{References}

1. Gensler MB. Silverberg's principles and practice of surgical pathology and cytopathology, sinonasal, and nasopharyngeal surgical pathology. Silverberg SG. 4th ed Philadelphia Churchill Livingstone Elsevier: 2006; 793-811

2. Settipane GA. Epidemiology of nasal polyps. Allergy Asthma 1996; 17(5):231-6

3. Garg D, Mathur K. Clinicopathological Study of Space Occupying Lesions of Nasal Cavity, Paranasal Sinuses, and Nasopharynx. Journal of Clinical and Diagnostic Research 2014; 8(11):4-7

4. Gupta AD, Ghosh RN, Mukherjee C. Nasal polyps Histopathological spectrum. Indian J Otolaryngol Head Neck Surg. 1997; 49(1):32-7

5. Maheshwari A, Bansal A. Clinicopathological spectrum of sinonasal masses: a tertiary care hospital experience. International Journal of Otorhinolaryngology and Head and Neck Surgery 2017; 3(4):1015-9

6. Bakari A, Afolabi OA, Adoga AA, Kodiya A M, Ahmad B M Clinicopathological profile of sinonasal masses: An experience in National Ear care center. BMC Res Notes 2010; 3:186

7. Singh SG, Qureshi S, Jain L, Jadia S, Sharma S. Presentation of Lesions of Nose and Paranasal Sinuses at a Tertiary Care Center in Central India. Indian J Otolaryngol Head Neck Surg. 2018; 70(2): 284-9

8. Gupta R, Moupachi SS, Poorey VK. Sinonasal Masses: A Retrospective Analysis. Indian J Otolaryngol Head Neck Surg. 2013; 65(1):52-6

9. Zafar U, Khan N, Afroz N, Hasan SA. Clinicopathological study of non-neoplastic lesions of nasal cavity and paranasal sinuses. Indian Journal Pathology Microbiol. 2008; 51(1):26-9

10. Kale SU, Mohite U, Drake-Lee AB. Clinical and histopathological correlation of nasal polyp: are there any surprises? Clin Otolaryngol Allied Sci. 2001; 26(4):321-3

11. Diamantopoulus IO, Jones NS, Lowe J. All nasal polyps need histopathological examination: an audit-based appraisal of clinical practice. J Laryngol Otol. 2000; 114(10):755-9

12. Sarkar S, Saha R, Das M, Sardar S. Sinonasal polyps: A diagnostic challenge. Bengal Journal of Otolaryngology and Head Neck Surgery 2019; 27(3): 243-50. 